\title{
Importance of Bevacizumab Maintenance Following Combination Chemotherapy in Human Non-small Cell Lung Cancer Xenograft Models
}

\author{
NOBUYUKI ISHIKURA, MIEKO YANAGISAWA, MARIKO NOGUCHI-SASAKI, TOSHIKI IWAI, \\ KEIGO YOROZU, MITSUE KURASAWA, MASAMICHI SUGIMOTO and KANAME YAMAMOTO \\ Product Research Department, Chugai Pharmaceutical Co., Ltd., Kamakura, Japan
}

\begin{abstract}
Background: Bevacizumab in combination with chemotherapeutics has shown significant survival benefit in clinical studies in patients with non-small cell lung cancer (NSCLC). Since bevacizumab was administered as standard treatment until disease progression, the importance of bevacizumab during the maintenance phase was not prospectively investigated in these studies. Materials and Methods: Three human NSCLC cell line xenograft models were used to investigate antitumor effect of bevacizumab and tumor microvessel density (MVD) was analyzed. Results: In A549 and NCI-H292 models, bevacizumab maintenance following combination with paclitaxel exhibited stronger tumor suppression than its absence. In an NCI-H292 model, bevacizumab maintenance continuously inhibited increase of MVD. In an NCI-H2228 model following induction treatment with pemetrexed and bevacizumab, maintenance with pemetrexed plus bevacizumab, had stronger efficacy than pemetrexed alone and led to lower MVD and level of thymidylate synthase. Conclusion: Continuous suppression of angiogenesis by bevacizumab may contribute to the superior efficacy of maintenance treatment containing bevacizumab.
\end{abstract}

Non-small cell lung cancer (NSCLC) accounts for 80-85\% of all lung cancers (1). Modalities for the treatment of advanced NSCLC following induction chemotherapy or radiotherapy have until recently been limited, but maintenance therapy is attracting attention as a new

Correspondence to: Nobuyuki Ishikura, Product Research Department, Chugai Pharmaceutical Co., Ltd. 200 Kajiwara, Kamakura, Kanagawa 247-8530, Japan. Tel: +81-467-47-6251, Fax: +81-467-45-6782, e-mail: ishikuranby@ chugai-pharm.co.jp

Key Words: Bevacizumab, paclitaxel, pemetrexed, maintenance therapy, angiogenesis, thymidylate synthase, non-small cell lung cancer. therapeutic strategy for NSCLC and several maintenance therapies that benefit these patients have become available.

The ECOG4599 study demonstrated that overall survival (OS) and progression-free survival (PFS) were significantly prolonged in patients with locally advanced metastatic or recurrent NSCLC who received bevacizumab maintenance following induction therapy with carboplatin and paclitaxel plus bevacizumab compared to patients who received induction therapy with carboplatin plus paclitaxel without maintenance therapy (2). The AVAPERL study demonstrated that PFS was significantly prolonged in patients with advanced NSCLC who received maintenance therapy with bevacizumab plus pemetrexed compared to those who received maintenance therapy with bevacizumab alone following induction therapy with cisplatin and pemetrexed plus bevacizumab (3). However, neither of these clinical studies drew the following two comparisons. One is the comparison of giving or not giving bevacizumab maintenance therapy following induction therapy with carboplatin and paclitaxel plus bevacizumab. The other is the comparison of giving bevacizumab plus pemetrexed maintenance with giving pemetrexed alone for maintenance following induction therapy with cisplatin and pemetrexed plus bevacizumab.

Bevacizumab is a humanized monoclonal antibody against vascular endothelial cell growth factor (VEGF) that inhibits VEGF-mediated angiogenesis in many types of cancer. Pemetrexed is a folate antagonist that exhibits an antitumor effect by inhibiting several enzymes involved in the metabolism of folic acid, such as thymidylate synthase (TYMS), dihydrofolate reductase and glycinamide ribonucleotide formyltransferase (4). Both preclinical and clinical studies have reported that TYMS expression level is a determinant of pemetrexed sensitivity in NSCLC (5-7).

In the present study, we used human NSCLC xenograft models to investigate the antitumor effect of maintenance treatment with bevacizumab alone compared with bevacizumab plus a chemotherapeutic agent following induction treatment with regimens including bevacizumab. 


\section{Materials and Methods}

Antitumor agents. Bevacizumab was provided by Hoffmann-La Roche AG (Basel, Switzerland) as a solution and diluted with saline. Human immunoglobulin $\mathrm{G}$ (HuIgG) was purchased from MP Biomedicals, Inc. (Aurora, OH, USA) and was reconstituted with water and diluted with saline. Paclitaxel was purchased from Wako Pure Chemical Industries (Tokyo, Japan). Pemetrexed disodium was purchased from AK Scientific (Union City, CA, USA).

Animals. Male, 4- or 5-week-old BALB-nu/nu mice were purchased from Charles River Laboratories Japan, Inc. (Tokyo, Japan). Male, 6week-old C.B-17/Icr-SCID Jcl severe combined immune-deficient (SCID) mice were purchased from CLEA Japan, Inc. (Tokyo, Japan). All animals were housed in a specific pathogen-free environment under controlled conditions (temperature, $20-26^{\circ} \mathrm{C}$; humidity, $35-75 \%$; light/dark cycle, $12 / 12 \mathrm{~h}$ ) and were allowed to acclimatize and recover from shipping-related stress for more than 6 days prior to the study. Chlorinated water and irradiated food were provided ad libitum. The health of the mice was monitored by daily observation. All animal experiments were reviewed and approved by the Institutional Animal Care and Use Committee at Chugai Pharmaceutical Co., Ltd. (Approval numbers:08-208, 09-424, 12-007).

Cell lines and culture conditions. Three human NSCLC cell lines were used in the present study: A549, with overexpression of both wild-type epidermal growth factor receptor $(E G F R)$ and human EGFR-related 2 (HER2) (8); NCI-H292, with wild-type EGFR overexpression (9); and NCI-H2228, with wild-type EGFR and echinoderm microtubule associated protein like 4 (EML4)anaplastic lymphoma kinase (ALK) fusion. All cell lines were obtained from the American Type Culture Collection (Manassas, VA, USA). A549 cells were maintained in F12-K Nutrient Mixture (Invitrogen, Carlsbad, CA, USA) supplemented with $10 \%$ fetal bovine serum (FBS; Japan Bioserum, Hiroshima, Japan). NCI-H292 and NCI-H2228 cells were maintained in RPMI-1640 medium (Sigma-Aldrich, St. Louis, MO, USA) supplemented with $10 \%$ FBS (Bovogen Biologicals, Melbourne, Australia).

In vivo tumor growth inhibition studies. Each BALB-nu/nu mouse was inoculated subcutaneously into the right flank with either A549 $\left(5 \times 10^{6}\right.$ cells $)$ or NCI-H292 $\left(5 \times 10^{6}\right.$ cells $)$. Each SCID mouse was inoculated subcutaneously into the right flank with NCI-H2228 $\left(1 \times 10^{7}\right.$ cells $)$. Several weeks after tumor inoculation, the mice were randomly allocated to control and induction treatment groups (day 1).

As the induction treatment in the A549 and NCI-H292 xenograft models, HuIgG or bevacizumab $(5 \mathrm{mg} / \mathrm{kg}$, the maximum effective dose; intraperitoneally) and paclitaxel vehicle (5\% ethanol-5\% Cremophor EL-saline; intravenously) or paclitaxel $(20 \mathrm{mg} / \mathrm{kg}$; the maximum tolerated dose in the A549 xenograft model; intravenously) were administered on days 1,8 and 15. On day 22, mice treated with bevacizumab plus paclitaxel were re-randomized into control and bevacizumab maintenance groups. At rerandomization of the A549 xenograft mice, four mice were excluded to reduce the variability in tumor volume and body weight. As maintenance treatment, HuIgG or bevacizumab was administered weekly until day 78 in the A549 xenograft model and until day 50 in the NCI-H292 xenograft model. Tumor volume (TV) was measured twice a week. The antitumor activity was evaluated by TV on day 85 (A549 model) or day 57 (NCI-H292 model).
As the induction treatment in the NCI-H2228 xenograft model, $\mathrm{HuIgG}$ or bevacizumab (5 mg/kg; maximum effective dose) and vehicle (saline) or pemetrexed $(400 \mathrm{mg} / \mathrm{kg}$; maximum effective dose) were administered intraperitoneally on days 1,8 and 15 . On day 22 , the mice treated with bevacizumab plus pemetrexed were rerandomized into four groups (control, bevacizumab, pemetrexed and bevacizumab plus pemetrexed). As the maintenance treatment, HuIgG or bevacizumab and vehicle or pemetrexed were administered intraperitoneally every week until day 78 . TV was measured twice a week. The antitumor activity was evaluated by TV on day 85 .

$\mathrm{TV}$ was estimated from the equation $\mathrm{TV}=a b^{2} / 2$, where $\mathrm{a}$ and $\mathrm{b}$ were tumor length and width, respectively.

Quantification of microvessel density in tumor tissues. Microvessel density (MVD) in tumor tissues was evaluated by immunohistochemical staining of cluster of differentiation 31 (CD31). Tumor samples were collected on indicated days. Immunohistochemical staining was conducted as described previously (10). MVD was calculated as the percentage ratio of the CD31-positive staining area to the total observation area in the viable region. Three to six fields per section were analyzed, excluding necrotic areas. Positively stained areas were calculated using imaging analysis software (WinROOF; Mitani Corporation, Fukui, Japan). Rat anti-mouse monoclonal antibody to CD31 (clone MEC 13.3) was purchased from BD Biosciences (San Jose, NJ, USA).

Western blotting. Tumor tissues taken from xenografts and stored at $-80^{\circ} \mathrm{C}$ were homogenized with Cell Lysis Buffer (Cell Signaling Technology, Danvers, MA, USA) with Complete Protease Inhibitor Cocktail Tablets (Roche, Basel, Switzerland). The homogenate was centrifuged at $9,100 \times g$ for $20 \mathrm{~min}$. The resultant supernatant was used for the assays. Protein concentration of the supernatant was quantified by using a Direct Detect spectrometer (Merck Millipore, Darmstadt, Germany). Cell lysates (5 $\mu \mathrm{g}$ protein/lane) were electrophoresed on sodium dodecyl sulfate-polyacrylamide gel and transferred to a polyvinylidene difluoride membrane using an iBlot Gel Transfer Device (Invitrogen). The membrane was blocked with Blocking One (Nacalai Tesque, Kyoto, Japan) and was primarily treated with antibody to TYMS (Cell Signaling Technology) and antibody to $\beta$-actin (Sigma-Aldrich) for immunoblotting. ECL Prime Western Blotting Detection Reagents and ImageQuant 400 (GE Healthcare Life Sciences, Little Chalfont, UK) were used for detection. ImageQuant TL Software (GE Healthcare Life Sciences) was used to digitize the strength of the bands.

Statistical analysis. The Wilcoxon test was used, with $p<0.05$ being considered statistically significant. Statistical analyses were carried out using the SAS preclinical package (SAS Institute, Inc., Tokyo, Japan).

\section{Results}

Antitumor activity of bevacizumab maintenance treatment following bevacizumab plus paclitaxel induction treatment. In the A549 xenograft model, TV in the bevacizumab plus paclitaxel group on day 22 was significantly lower than that in the control group, indicating that tumor growth was completely inhibited during the induction treatment (Figure 1A). TV on day 85 during maintenance treatment was significantly higher in the control group, indicating that 

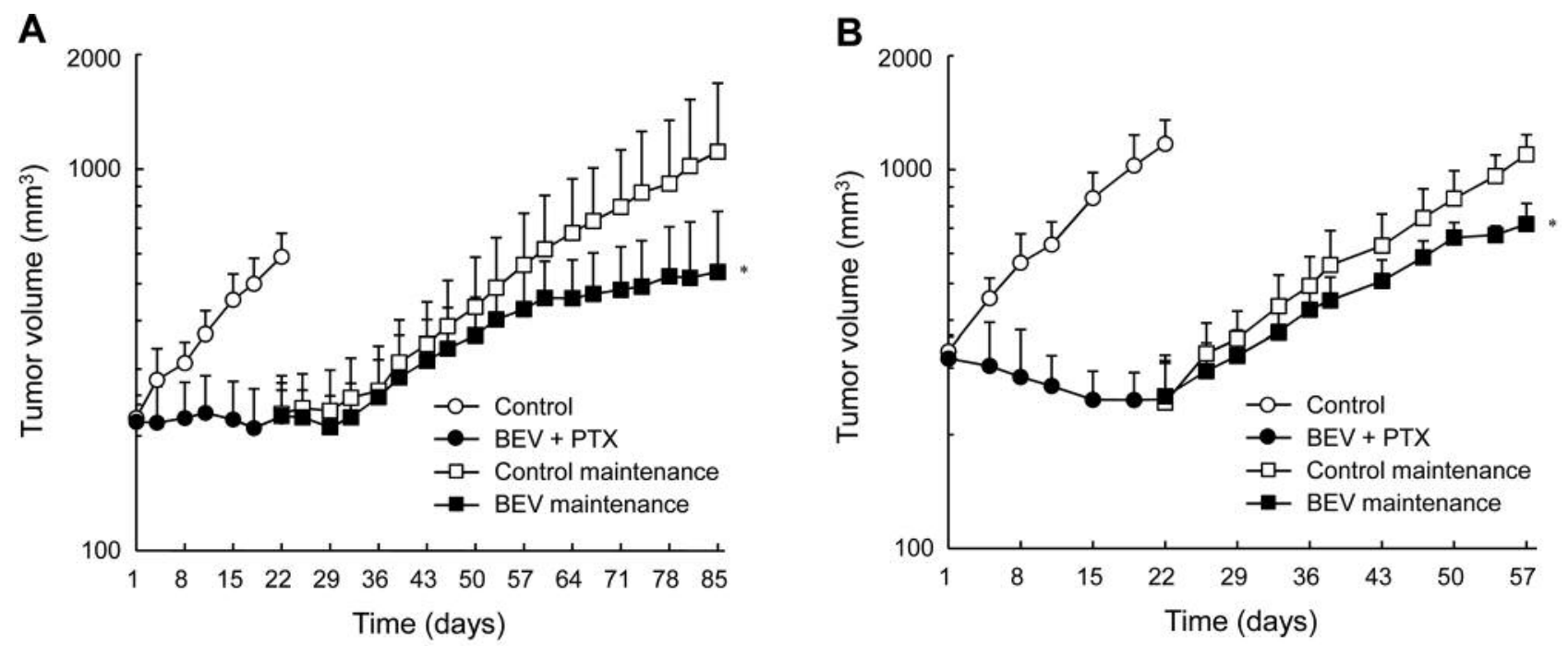

Figure 1. Antitumor activity of bevacizumab (BEV) maintenance following BEV plus paclitaxel (PTX) induction treatment in A549 and NCI-H292 xenograft models. A: Mice bearing A549 tumors were randomly divided into a control group $(n=8)$ and a BEV $+P T X$ group $(n=36)$ and treated with vehicle or drugs on days 1,8 and 15 . On day 22, mice treated with BEV+PTX were re-randomized into a control maintenance group ( $n=16)$ and a BEV maintenance group $(n=16)$ and treated with vehicle or BEV weekly until day 78. B: Mice bearing NCI-H292 tumors were similarly randomly divided into a control group $(n=6)$ and a BEV+PTX group $(n=12)$ and treated with vehicle or drugs on days 1,8 and 15. On day 22, mice treated with BEV+PTX were re-randomized into a control maintenance group $(n=6)$ and a BEV maintenance group $(n=6)$ and treated with vehicle or BEV weekly until day 50. The dosage of BEV and PTX was $5 \mathrm{mg} / \mathrm{kg}$ and $20 \mathrm{mg} / \mathrm{kg}$, respectively. Data points represent the mean $+S D$ tumor volume (mm $\left.{ }^{3}\right)$. *Statistically significant differences at $p<0.05 \mathrm{vs}$. control maintenance group by Wilcoxon test.

bevacizumab exhibited significantly strong antitumor activity compared to control $(p<0.05)$.

In the NCI-H292 xenograft model, TV in the bevacizumab plus paclitaxel group on day 22 was also significantly lower than that in the control group, indicating that bevacizumab plus paclitaxel completely inhibited tumor growth during the induction treatment (Figure 1B) and TV comparison on day 57 indicated that bevacizumab exhibited a significantly strong antitumor activity compared to control by maintenance treatment $(p<0.05)$.

Anti-angiogenic activity of bevacizumab maintenance treatment following bevacizumab plus paclitaxel induction treatment in the NCI-H292 xenograft model. The MVD in NCI-H292 tumor tissues was evaluated from specimens obtained on days 22, 36 and 57. MVD on day 22 was significantly inhibited during induction treatment bevacizumab plus paclitaxel $(p<0.05)$ (Figure 2). Moreover, MVD in the bevacizumab maintenance group on days 36 and 57 did not differ but was significantly lower than that of the control, indicating that bevacizumab continuously inhibited MVD increase during maintenance treatment.

Antitumor activity of bevacizumab plus pemetrexed maintenance treatment following bevacizumab plus pemetrexed induction treatment in the NCI-H2228 xenograft model. Bevacizumab plus pemetrexed completely inhibited the tumor growth during the induction treatment (Figure 3). The TV in each treatment group on day 85 was significantly reduced $(p<0.05)$ compared with the control group. Of note, bevacizumab plus pemetrexed completely inhibited tumor growth not only during induction but also during maintenance treatment, with significantly stronger antitumor activity than with monotherapy with bevacizumab $(p<0.05)$ or pemetrexed $(p<0.05)$.

Anti-angiogenic activity of bevacizumab plus pemetrexed maintenance treatment following bevacizumab plus pemetrexed induction treatment in the NCI-H2228 xenograft model. MVD in NCI-H2228 tumor tissues on day 85 in the bevacizumab and bevacizumab plus pemetrexed maintenance groups was significantly lower $(p<0.05)$ than that in the control and pemetrexed maintenance groups. There was no statistical difference in MVD between the groups treated with bevacizumab alone or with bevacizumab plus pemetrexed maintenance (Figure 4).

TYMS expression level in the NCI-H2228 xenograft model. TYMS expression in NCI-H2228 tumor tissue was evaluated by western blotting on samples obtained on day 85. TYMS expression was significantly lower $(p<0.05)$ in the bevacizumab and bevacizumab plus pemetrexed maintenance 
A

\section{Control}

[day 22]

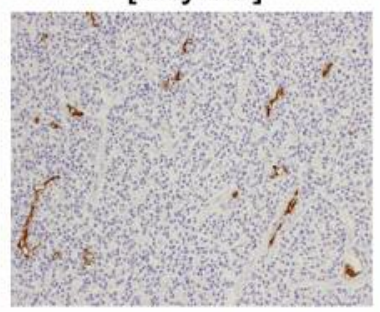

BEV maintenance [day 36]

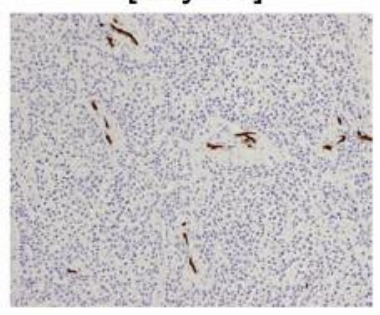

$\mathrm{BEV}+\mathrm{PTX}$

[day 22]

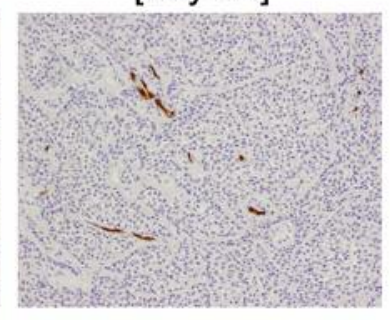

BEV maintenance

[day 57]

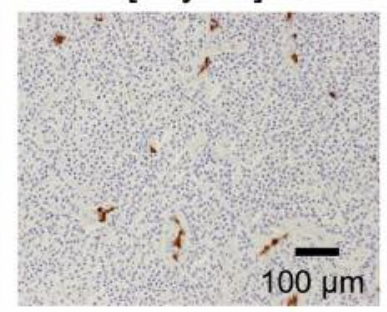

B

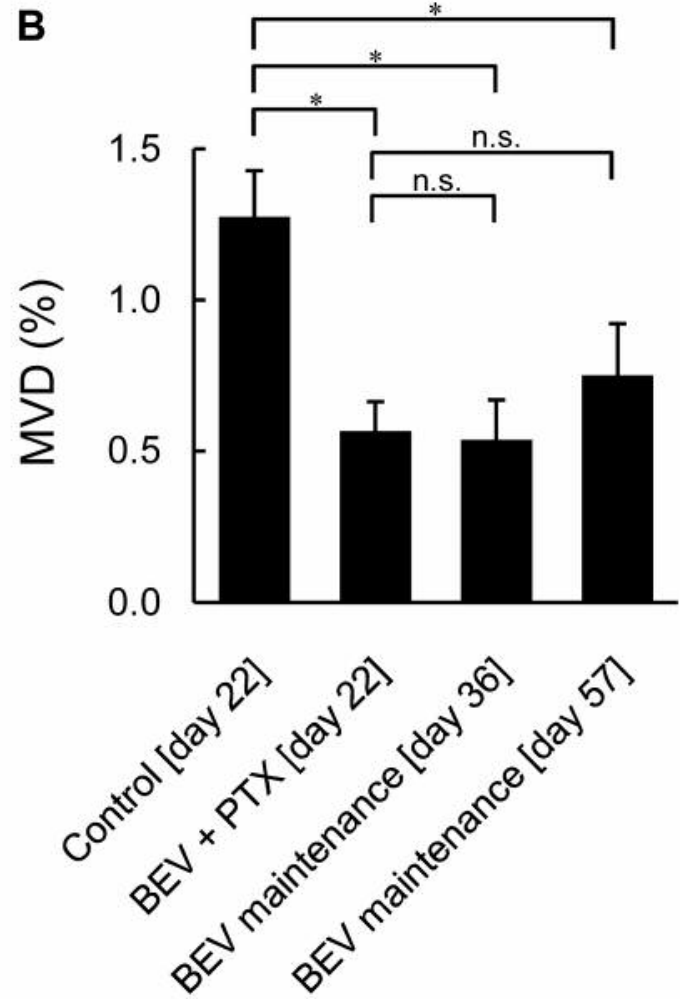

Figure 2. Anti-angiogenic effect of bevacizumab (BEV) maintenance following BEV plus paclitaxel (PTX) induction treatment in NCI-H292 xenograft model. A: Tumor microvessels stained immunohistochemically with antibody to CD31 on the indicated days. B: The microvessel density (MVD) in tumor tissue was determined by calculating the ratio of CD31-positive area to the total observed area. Data represent the mean+SD ( $n=6)$. *Statistically significant difference $(p<0.05)$ by Wilcoxon test. $n . s .:$ Non-significant $(p \geq 0.05)$ difference.

groups than that in the control and pemetrexed maintenance groups. No significant decrease in TYMS expression was observed in the pemetrexed group as compared with that in the control group (Figure 5).

\section{Discussion}

The present study was conducted to respond to two clinical questions regarding maintenance treatment with bevacizumab in advanced NSCLC. The first question arises from the study reported by Sandler et al. (ECOG4599 study) (2) concerning the requirement of bevacizumab maintenance following induction therapy with carboplatin and paclitaxel plus bevacizumab. The second arises from the study reported by Barlesi et al. (AVAPERL study) (3) and concerns the comparison between pemetrexed with and without bevacizumab in maintenance treatment following induction therapy with cisplatin and pemetrexed plus bevacizumab. To this end, experiments were carried out using mouse xenograft models inoculated with human NSCLC cell lines.
Firstly, the antitumor effect of the treatment regimen consisting of bevacizumab maintenance treatment following paclitaxel plus bevacizumab induction treatment was compared with the regimen without bevacizumab maintenance treatment following the same induction treatment. As a result, it was shown that the regimen with bevacizumab maintenance exhibited a stronger antitumor effect than the regimen without bevacizumab maintenance in two xenograft models, A549 and NCI-H292 (Figure 1). These results suggest that to produce superior efficacy, bevacizumab treatment is not only important during the induction phase in combination with paclitaxel, but also as maintenance therapy. Paclitaxel plus bevacizumab led to significantly reduced MVD in the NCI-H292 xenograft model on the last day (day 22) of the induction treatment compared with the control group (Figure 2). Subsequent treatment with bevacizumab maintained this effect in MVD throughout the study period until day 57 , i.e. no statistically significant difference was detected between the MVD of the paclitaxel plus bevacizumab group on day 22 and that of the bevacizumab maintenance group on day 57 (Figure 2). These 


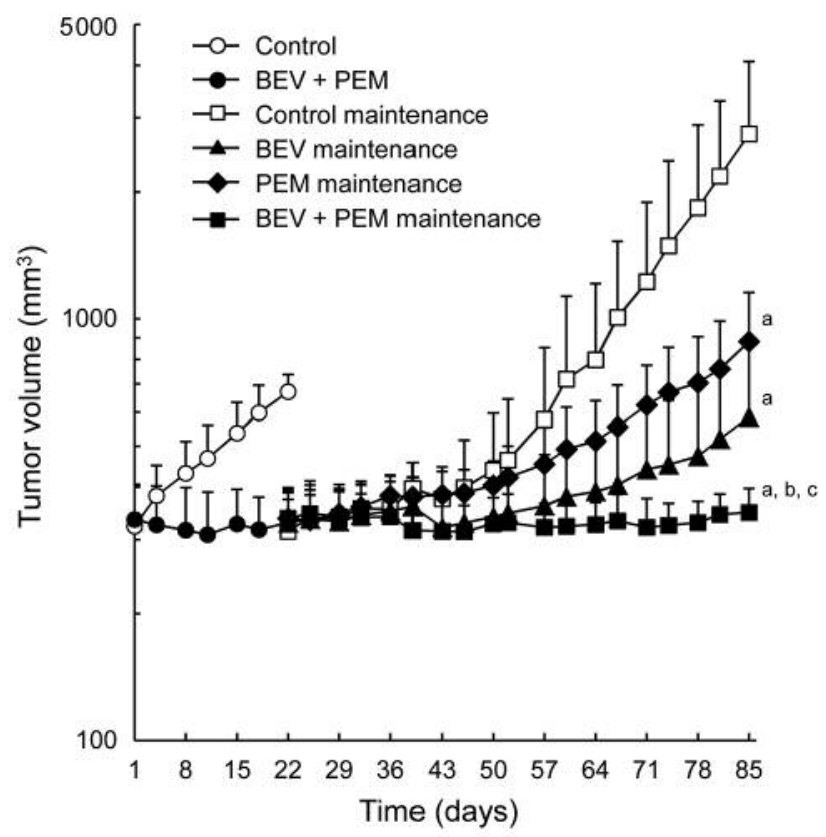

Figure 3. Antitumor activity of bevacizumab (BEV) plus pemetrexed (PEM) maintenance following BEV plus PEM induction treatment in NCI-H2228 xenograft model. Mice bearing NCI-H2228 tumors were randomly divided into a control group $(n=5)$ and a BEV+PEM group $(n=28)$ and treated with vehicle or drugs on days 1,8 and 15. On day 22 , mice treated with $B E V+P E M$ were re-randomized into four groups ( $n=7$ per group): control maintenance, BEV maintenance, PEM maintenance and BEV plus PEM maintenance groups. Mice were treated with vehicle, BEV with/without PEM weekly until day 78 . The dosage of BEV and PEM was $5 \mathrm{mg} / \mathrm{kg}$ and $400 \mathrm{mg} / \mathrm{kg}$, respectively. Data points represent the mean $+S D$ tumor volume $\left(\mathrm{mm}^{3}\right)$. Statistically significant different at $p<0.05 \mathrm{vs}$. ${ }^{a}$ control maintenance, ${ }^{b} B E V$ maintenance, and CPEM maintenance by Wilcoxon test.

results indicate that bevacizumab maintenance following bevacizumab plus paclitaxel induction is able to exert a continuous anti angiogenic effect in this model.

Secondly, inspired by the study reported by Barlesi et al. (AVAPERL study) (3), we investigated the antitumor effect of the treatment regimen consisting of bevacizumab with/without pemetrexed maintenance following bevacizumab plus pemetrexed induction treatment in an NCI-H2228 xenograft model. With regard to pemetrexed maintenance, the PARAMOUNT study demonstrated significant prolongation of OS in the pemetrexed maintenance arm compared with the best supportive care arm following induction therapy with pemetrexed plus cisplatin (11). Although the AVAPERL study demonstrated the superiority of maintenance with bevacizumab plus pemetrexed compared with that of bevacizumab alone, comparison between maintenance with bevacizumab plus pemetrexed and pemetrexed alone was not investigated. Recently, a phase II study was conducted comparing bevacizumab plus pemetrexed maintenance therapy and pemetrexed maintenance therapy following induction therapy with carboplatin and pemetrexed plus bevacizumab in patients with advanced NSCLC (12). In this phase II study, the bevacizumab plus pemetrexed maintenance arm showed longer median PFS than the pemetrexed maintenance arm; however, the difference was not statistically significant. The authors pointed out the limitations of their study, which included insufficient statistical power to detect differences due to a limited number of patients and the necessity of further comparative studies. In accordance with the results of the AVAPERL study, our study showed that the regimen of maintenance with bevacizumab plus pemetrexed exhibited a significantly stronger antitumor effect compared with maintenance of bevacizumab alone and compared with maintenance of pemetrexed alone (Figure 3). These results show the significance of continuing bevacizumab together with pemetrexed in the maintenance phase in order to achieve superior efficacy following bevacizumab plus pemetrexed induction treatment.

To investigate the mechanism by which bevacizumab with pemetrexed maintains an antitumor effect after induction treatment, the MVD in NCI-H2228 tumors was analyzed. Compared with the group treated with maintenance of pemetrexed alone, the MVD was significantly lower in group treated with bevacizumab plus pemetrexed maintenance as well as in that given bevacizumab alone. The bevacizumab-induced decrease in MVD was not affected by pemetrexed (Figure 4). Furthermore, TYMS expression level was significantly lower in the group receiving bevacizumab alone or bevacizumab plus pemetrexed as maintenance treatments than in the control group and that treated with maintenance of pemetrexed alone (Figure 5). Takezawa et al. showed that the level of TYMS is a determinant of pemetrexed sensitivity in NSCLC by forced overexpression of TYMS and its specific down-regulation by small interfering RNA in NSCLC cell lines (5). Indeed, patients with NSCLC with lower tumor TYMS expression exhibited longer PFS and OS by pemetrexed therapy in a few studies (5-7). Therefore, in addition to the reduction of angiogenesis, the decrease in TYMS by continuous administration of bevacizumab may also contribute to the strong antitumor effect of maintenance treatment of bevacizumab plus pemetrexed following the same as induction treatment. The precise mechanism by which bevacizumab reduces the TYMS level remains to be elucidated.

In conclusion, the present study showed that (i) bevacizumab maintenance treatment following bevacizumab plus paclitaxel induction treatment produced a superior antitumor effect compared with no maintenance and (ii) bevacizumab plus pemetrexed maintenance treatment following bevacizumab plus pemetrexed induction treatment had a superior antitumor effect compared with maintenance pemetrexed alone following the same induction treatment. 
A

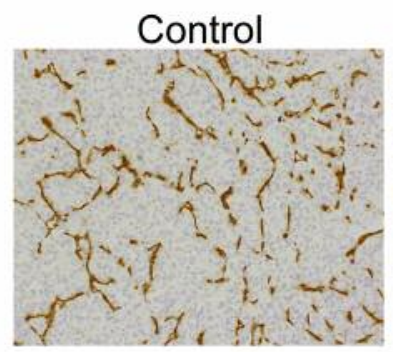

PEM

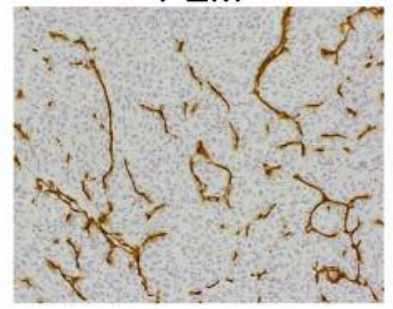

B

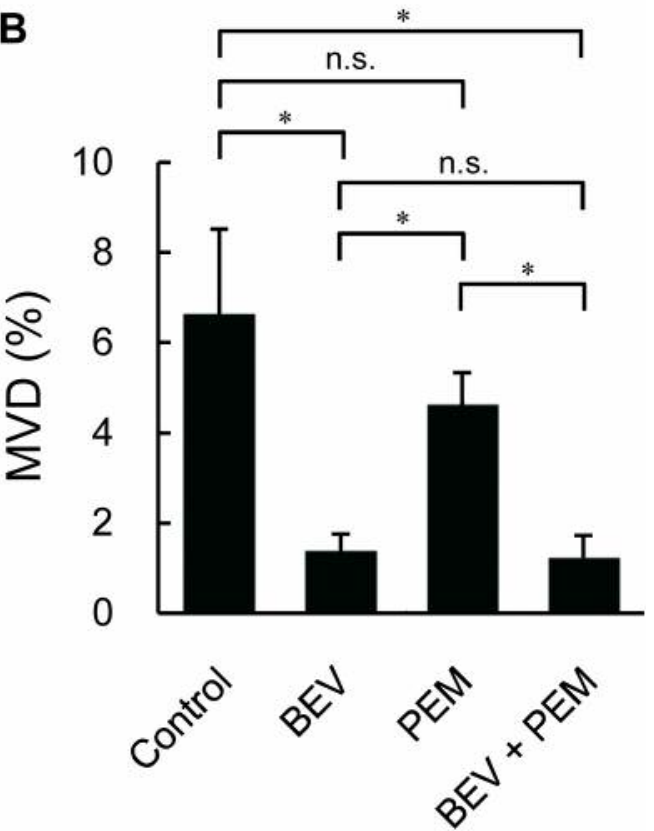

Figure 4. Anti-angiogenic activity of bevacizumab (BEV) plus pemetrexed (PEM) maintenance following BEV plus PEM induction treatment in NCIH2228 xenograft model. A: Tumor microvessels stained immunohistochemically with antibody to CD31 on day 85. B: The microvessel density (MVD) in tumor tissue was determined by calculating the ratio of the CD31-positive area to the total observed area. Data represent the mean+SD ( $n=6$ 7). *Statistically significant difference $(p<0.05)$ by Wilcoxon test. $n . s .:$ Non-significant $(p \geq 0.05)$ difference.

A

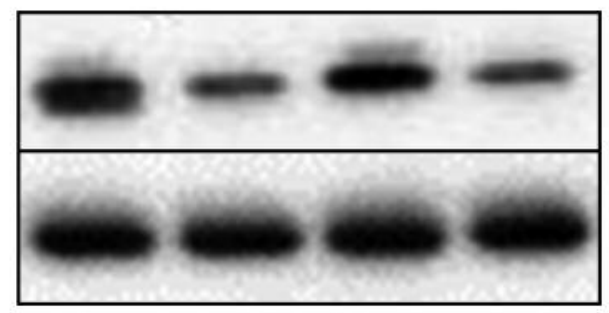<smiles>C1=C[Co][CH-]1</smiles>

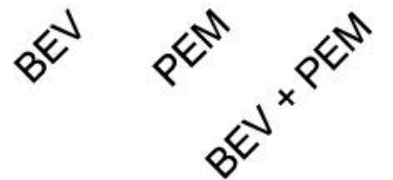

B

TYMS

$\beta$-Actin
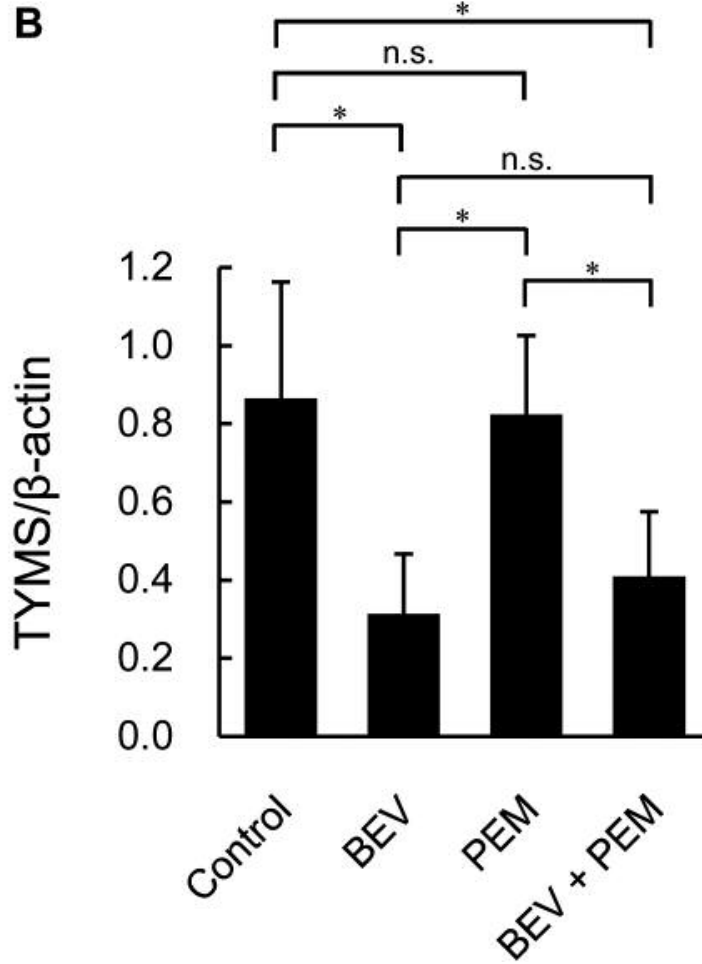

Figure 5. Thymidylate synthase (TYMS) expression level in NCI-H2228 xenografts treated with bevacizumab (BEV) plus pemetrexed (PEM) maintenance following BEV plus PEM induction treatment. A: TYMS expression in tumor tissue was analyzed by western blotting with antibody to TYMS on day 85. B: The TYMS expression level was determined by calculating the ratio of TYMS/ $\beta$-actin. Data represent the mean $+S D(n=6)$. *Statistically significant difference $(p<0.05)$ by Wilcoxon test. n.s.: Non-significant $(p \geq 0.05)$ difference. 
Both of these maintenance treatments with bevacizumab resulted in a continuous reduction of MVD, which suggests it is the main mechanism underlying the superior efficacy in maintenance treatments that include bevacizumab. These results suggest that the continuation of bevacizumab as a maintenance therapy after induction treatment including bevacizumab is beneficial for patients with advanced NSCLC, regardless of combined chemotherapy.

\section{Conflicts of Interest}

All Authors are employees of Chugai Pharmaceutical Co., Ltd.

\section{Acknowledgements}

The Authors thank Dr. Kaori Fujimoto-Ouchi for her advice on experimental design; Dr. Yoichiro Moriya for his help in article preparation; and Ms. Masako Miyazaki, Ms. Kumiko Kondo and Ms. Hiromi Sawamura for their excellent technical assistance.

\section{References}

1 Maione P, Rossi A, Sacco PC, Bareschino MA, Schettino C, Ferrara ML, Falanga M, Ambrosio R and Gridelli C: Treating advanced non-small cell lung cancer in the elderly. Ther Adv Med Oncol 2: 251-260, 2010.

2 Sandler A, Gray R, Perry MC, Brahmer J, Schiller JH, Dowlati A, Lilenbaum R and Johnson DH: Paclitaxel-carboplatin alone or with bevacizumab for non-small-cell lung cancer. $\mathrm{N}$ Engl $\mathrm{J}$ Med 355: 2542-2550, 2006.

3 Barlesi F, Scherpereel A, Rittmeyer A, Pazzola A, Ferrer Tur N, Kim JH, Ahn MJ, Aerts JG, Gorbunova V, Vikstrom A, Wong EK, Perez-Moreno P, Mitchell L and Groen HJ: Randomized phase III trial of maintenance bevacizumab with or without pemetrexed after first-line induction with bevacizumab, cisplatin and pemetrexed in advanced nonsquamous non-small-cell lung cancer: AVAPERL (MO22089). J Clin Oncol 31: 3004-3011, 2013.

4 Shimizu T, Nakanishi Y, Nakagawa Y, Tsujino I, Takahashi N, Nemoto $\mathrm{N}$ and Hashimoto $\mathrm{S}$ : Association between expression of thymidylate synthase, dihydrofolate reductase and glycinamide ribonucleotide formyltransferase and efficacy of pemetrexed in advanced non-small cell lung cancer. Anticancer Res 32: 45894596, 2012.

5 Takezawa K, Okamoto I, Okamoto W, Takeda M, Sakai K, Tsukioka S, Kuwata K, Yamaguchi H, Nishio K and Nakagawa $\mathrm{K}$ : Thymidylate synthase as a determinant of pemetrexed sensitivity in non-small cell lung cancer. Br J Cancer 104: 1594$1601,2011$.
6 Christoph DC, Asuncion BR, Hassan B, Tran C, Maltzman JD, O'Shannessy DJ, Wynes MW, Gauler TC, Wohlschlaeger J, Hoiczyk M, Schuler M, Eberhardt WE and Hirsch FR: Significance of folate receptor alpha and thymidylate synthase protein expression in patients with non-small-cell lung cancer treated with pemetrexed. J Thorac Oncol 8: 19-30, 2013.

7 Nicolson MC, Fennell DA, Ferry D, O’Byrne K, Shah R, Potter V, Skailes G, Upadhyay S, Taylor P andre V, Nguyen TS, Myrand SP, Visseren-Grul C, Das M and Kerr KM: Thymidylate synthase expression and outcome of patients receiving pemetrexed for advanced nonsquamous non-small-cell lung cancer in a prospective blinded assessment phase II clinical trial. J Thorac Oncol 8: 930-939, 2013.

8 Diaz R, Nguewa PA, Parrondo R, Perez-Stable C, Manrique I, Redrado M, Catena R, Collantes M, Penuelas I, Diaz-Gonzalez JA and Calvo A: Antitumor and antiangiogenic effect of the dual EGFR and HER2 tyrosine kinase inhibitor lapatinib in a lung cancer model. BMC Cancer 10: 188, 2010.

9 Ono N, Yamazaki T, Tsukaguchi T, Fujii T, Sakata K, Suda A, Tsukuda T, Mio T, Ishii N, Kondoh O and Aoki Y: Enhanced antitumor activity of erlotinib in combination with the HSP90 inhibitor CH5164840 against non-small-cell lung cancer. Cancer Sci 104: 1346-1352, 2013.

10 Yanagisawa M, Yorozu K, Kurasawa M, Nakano K, Furugaki K, Yamashita Y, Mori K and Fujimoto-Ouchi K: Bevacizumab improves the delivery and efficacy of paclitaxel. Anticancer Drugs 21: 687-694, 2010.

11 Paz-Ares LG, de Marinis F, Dediu M, Thomas M, Pujol JL, Bidoli P, Molinier O, Sahoo TP, Laack E, Reck M, Corral J, Melemed S, John W, Chouaki N, Zimmermann AH, VisserenGrul C and Gridelli C: PARAMOUNT: Final overall survival results of the phase III study of maintenance pemetrexed versus placebo immediately after induction treatment with pemetrexed plus cisplatin for advanced nonsquamous non-small-cell lung cancer. J Clin Oncol 31: 2895-2902, 2013.

12 Karayama M, Inui N, Fujisawa T, Enomoto N, Nakamura Y, Kuroishi S, Yokomura K, Koshimizu N, Sato M, Toyoshima M, Shirai T, Masuda M, Yamada T, Imokawa $S$ and Suda T: Maintenance therapy with pemetrexed and bevacizumab versus pemetrexed monotherapy after induction therapy with carboplatin, pemetrexed and bevacizumab in patients with advanced non-squamous non small cell lung cancer. Eur J Cancer 58: 30-37, 2016.
Received November 19, 2016

Revised December 16, 2016

Accepted December 20, 2016 